\title{
3D Volumetric Analysis and Haptic Modeling for Preoperative Planning in Breast Reconstruction
}

\author{
Michael P Chae ${ }^{1,2,3}$, David J Hunter-Smith ${ }^{1,2,3}$, Alexandra Rizzitelli ${ }^{1,2,3}$, Robert T Spychal ${ }^{1,2,3}$, Warren Matthew Rozen ${ }^{1,2,3^{*}}$ \\ ${ }^{1}$ Department of Plastic and Reconstructive Surgery, Frankston Hospital, Peninsula Health, 2 Hastings Road, Frankston Victoria 3199, Australia \\ ${ }^{2}$ Department of Surgery, Monash University, Level 5, E Block, Monash Medical Centre, 246 Clayton Road, Clayton Victoria 3168, Australia \\ ${ }^{3}$ Monash University Plastic and Reconstructive Surgery Unit (Peninsula Clinical School), Peninsula Health, 2 Hastings Road, Frankston Victoria 3199, Australia
}

*Corresponding author: Warren M Rozen, Department of Plastic and Reconstructive Surgery, Frankston Hospital, Peninsula Health, 2 Hastings Road, Frankston Victoria 3199 Australia, Phone: +613 9784-8416; Fax: +613 9784-7568; Email: warrenrozen@hotmail.com

Received date: June 28, 2014, Accepted dateDecember 27, 2014, Published date:January 1, 2015

Copyright: (c) 2014 Warren M Rozen et al. This is an open-access article distributed under the terms of the Creative Commons Attribution License, which permits unrestricted use, distribution, and reproduction in any medium, provided the original author and source are credited.

\begin{abstract}
Background: Preoperative planning and imaging have shown improved clinical outcome in breast reconstructions. However, an accurate, objective method of volumetric analysis has eluded investigators in the past. Furthermore, scan data from the current imaging modality are limited by the two dimensional (2D) representation on a computer screen. The introduction of 3D haptic biomodeling has led to a more intuitive understanding of the relationship between anatomical structures.
\end{abstract}

Method:We describe an easy, reproducible, accessible approach to volumetric analysis of the breast, by 3Dprinting a haptic model from the scan data. This approach comprises use of a single computed tomography (CT) or magnetic resonance imaging (MRI) scan for volumetric analysis, which we use to compare to simpler estimation techniques, create software-generated 3D reconstructions, calculate and visualize volume differences, and produce biomodels of the breasts using a 3D printer for tactile appreciation of volume differential.

Results:Using the technique described, parenchymal volume was assessed and calculated using CT data. Two cases of breast asymmetry were utilized in a pictorial account of the technique, in which a volume difference of $116 \mathrm{~cm}^{3}$ and $124.61 \mathrm{~cm}^{3}$ respectively was calculated, aiding reconstructive planning.

Conclusion: Preoperative planning can improve aesthetic and clinical outcomes in breast reconstruction by achieving symmetry, reducing operative length and complications. We demonstrate that our technique of volumetric analysis and the production of 3D haptic biomodels will be a valuable addition to the current array of preoperative planning tools. Our findings warrant a further evaluation of the technique for correlation with clinical outcomes through trials.

Keywords: Volumetric analysis; Preoperative imaging; Planning; 3D printing; Model

\section{Introduction}

Autologous breast reconstruction with a perforator flap has benefited in recent times from preoperative assessment with appropriate imaging modalities [1]. Assessing the breast volume for, for example, comparison with the contralateral side would provide a valuable information about the difference in volume, suitable implant size and flap design [2]. However, an accurate, objective, reproducible method of volumetric analysis has not been identified in the literature yet. In the last decade or so, multiple evidences have established computed tomographic angiography (CTA) as the imaging of choice in perforator flap surgery for the selection of donor site, perforator and flap designing [3]. In recent times, the ability to three-dimensionally (3D) print haptic biomodels, which provides a superior mode of visualizing the spatial relationship between anatomical structures, has added an interesting dimension to the preoperative planning [4].

The knowledge of accurate breast volume can enable a reconstructive surgeon to devise the optimal size of the implant or a flap in order to achieve a symmetrical, satisfactory outcome. A significant number of manual techniques have been described in the literature without much avail, such as direct anthropomorphic measurement, techniques utilizing water displacement, paraffin, and casting. Hence, most reconstructive surgeons still rely on 2D imaging, such as computed tomography $(\mathrm{CT})$ and magnetic resonance imaging (MRI), and subjective visual assessment for evaluating breast volume, which varies in accuracy depending on the experience and ability of the surgeon. Recently, an objective method of volume measurement by $3 \mathrm{D}$ imaging has been reported [5,6]. 3D imaging utilizes a plain light source or a laser (i.e. stereophotogrammetry or laser scanning technology, respectively) to obtain a 3D surface model, from which a tissue volume is quantified. A caveat of $3 \mathrm{D}$ imaging exists in that it does not visualize the posterior surface of the breasts and hence, not account for the individual variances in the shape of the anterior chest wall potentially leading to miscalculations.

Currently, CTA is the most accurate imaging modality for the preoperative evaluation of the donor site for perforator and flap selection and for appraisal of the recipient vessels for free tissue transfer [3,7]. It is quick, can assess multiple perforators and donor sites at once, and produces images that are relatively easy for the 
surgeons to interpret [8]. Evidences demonstrate that CTA is associated with minimal donor site morbidity, reduced operative length, improved clinical outcome, and a decrease in complications $[9,10]$. In comparison to Doppler ultrasound, CTA is a superior option for identifying the perforator vascular anatomy and the newer magnetic resonance angiography (MRA) has not been explored and reported frequently enough to justify a widespread use despite its slow speed and high cost [11].

Well-established and routinely used in industrial design for decades, rapid prototyping (RP) and the $3 \mathrm{D}$ printing technology has recently become widely available for medical application $[4,12-15]$. CT or MRI scan data can be imported using an appropriate 3D software, such as Osirix (Pixmeo, Geneva, Switzerland), and is used to fabricate $3 \mathrm{D}$ haptic biomodels that are superior to the traditional 2D images for providing spatial anatomical relationships [16]. One of the major limitations has been for the physicians having to outsource this technology, which is associated with high cost and subsequent slow production speed. To this end, we have recently described a method of enabling 3D printing accessible as an affordable office-based tool for an everyday surgeon [2].

\section{Methods}

In this current study, we demonstrate a reproducible and affordable method of performing accurate volumetric analysis and fabricating $3 \mathrm{D}$ haptic biomodels for preoperative planning in breast reconstruction. We used a computer software, Osirix, to accurately estimate the breast volume from the routine $\mathrm{CT}$ scan and created $3 \mathrm{D}$ visualizations to $3 \mathrm{D}$ print models for appreciating the volume difference in a tactile manner. For our purpose, we illustrate a case of asymmetrical breast following a left breast implant.

\section{Technique}

A routine CTA is performed with the patient lying prone, back extended superiorly at 45 degrees, and bra off (Figure 1).

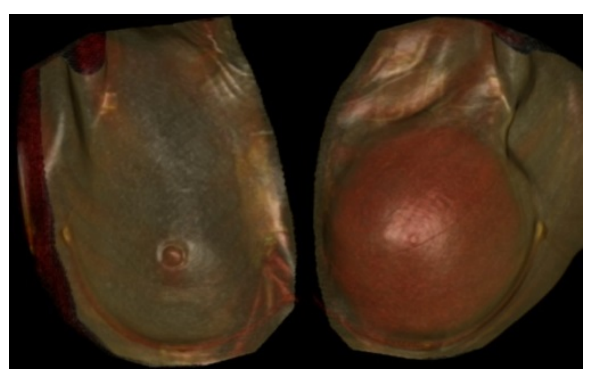

Figure 1: Surface-rendered three-dimensional (3D) reconstruction of a computed tomographic (CT) scan of the breasts, performed with Osirix (Pixmeo, Geneva, Switzerland) software. Reproduced with permission from: Chae MP, Hunter-Smith DJ, Spychal RT, Rozen WM. 3D Volumetric Analysis for Planning Breast Reconstructive Surgery. Breast Cancer Res Treat. 2014 June; [Epub ahead of Print].

This position ensures that the breasts are hanging loosely, subject to the gravity. Thin slices $(0.625 \mathrm{~cm})$ of the CTA are uploaded on to Osirix (Pixmeo, Geneva, Switzerland), a free third-party software. The breast volume is calculated by highlighting the breast parenchyma on each transverse slice using "closed polygon mouse" function and then, "compute volume" function (Figure 2).
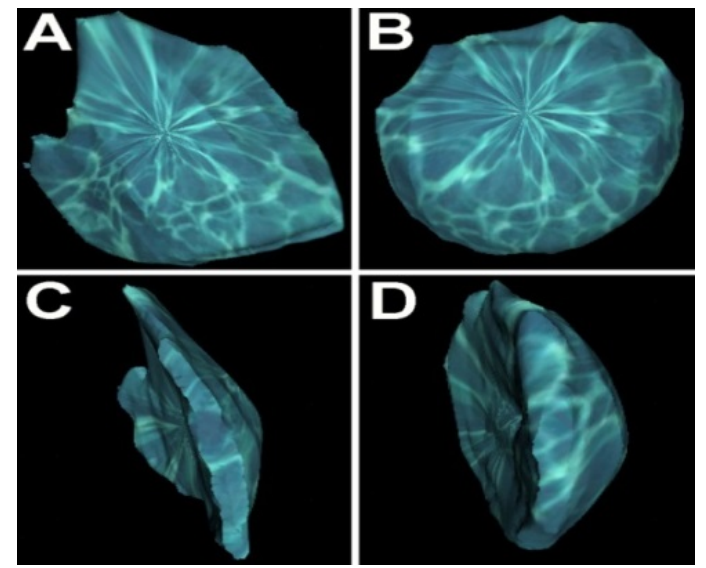

Figure 2: Three-dimensional analysis of the breast parenchymal volume, after selective cropping of the skin and chest wall from scan data, using volume-rendered three-dimensional (3D) reconstruction of a computed tomographic (CT) scan of the breasts (same patient as Figure 1) with Osirix (Pixmeo, Geneva, Switzerland) software: $\mathrm{A}=$ right breast, anterior view ; $\mathrm{B}=$ left breast, anterior view ; $\mathrm{C}=$ right breast, lateral view ; $\mathrm{D}=$ left breast, lateral view. Reproduced with permission from: Chae MP, Hunter-Smith DJ, Spychal RT, Rozen WM. 3D Volumetric Analysis for Planning Breast Reconstructive Surgery. Breast Cancer Res Treat. 2014 June; [Epub ahead of Print].

The attention is paid to exclude chest wall components into the calculation, such as the pectoralis major and minor. The calculated left breast volume was $403 \mathrm{~cm}^{3}$, the right breast $287 \mathrm{~cm}^{3}$, and hence, the difference was $116 \mathrm{~cm}^{3}$. Using recently developed 3D modeling techniques, we demonstrate a $3 \mathrm{D}$ visualization of the volume difference between the breasts. This provides additional information about the volume difference, such as the shape and contour. Firstly, the scan data is reconstructed into a $3 \mathrm{D}$ image using "volume rendering" function on Osirix. The breasts are isolated and other unnecessary structures, such as the arms, jaw, and abdomen, are removed from view using "scissor" function. Then, the isolated breasts are remodeled using "surface rendering" function. This 3D image is exported in Standard Tessellation Language (STL) file format, used universally in 3D modeling. Each breast is saved as separate STL files and then, loaded simultaneously on to Magics Software (Materialise, Leuven, Belgium) (Figure 3).

After the 3D images are aligned, a duplicate mirror image of the left breast is created. This image is translated and superimposed on to the right breast. The two images are subtracted from each other using "Boolean" function in Magics. The remaining "reverse image" is the physical representation of the volume difference. Enabled by a recent advancement in manufacturing 3D models using affordable $3 \mathrm{D}$ printers, we have fabricated a haptic model of the breasts from a routine CT scan data. These models can add an extra dimension, tactile feedback, to the appreciation of breast volume difference. 


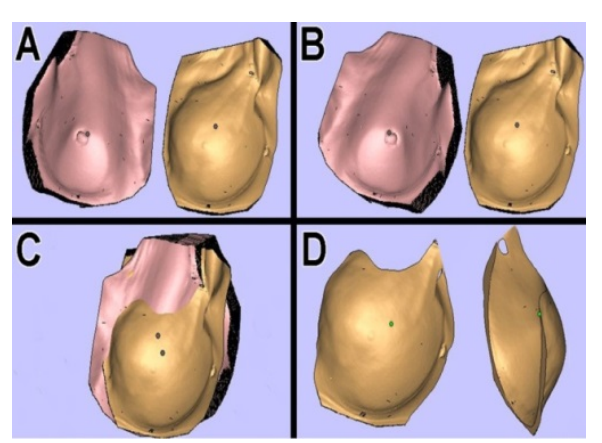

Figure 3: Production of a three-dimensional 'subtracted' volume difference between breasts (same patient as Figure 1), using volume-rendered CT data in Magics Software (Materialise, Leuven, Belgium). Volume-rendered computed tomography (CT) images are aligned (A), a mirror image of one breast is created to align both breasts unidirectionally (B), the two volumes superimposed (C), and the volumes are 'subtracted' from one another to produce the volume difference (D). Reproduced with permission from: Chae MP, Hunter-Smith DJ, Spychal RT, Rozen WM. 3D Volumetric Analysis for Planning Breast Reconstructive Surgery. Breast Cancer Res Treat. 2014 June; [Epub ahead of Print].

To achieve this, we imported the STL file of the breasts on to a 3D printer software, Cubify (3D Systems, Rock Hill, SC, USA), which renders the 3D image suitable for printing (Cube 2 printer; 3D Systems, Rock Hill, SC, USA) (Figure 4). We used white polylactic acid (PLA) filament and the print duration was 15 hours.

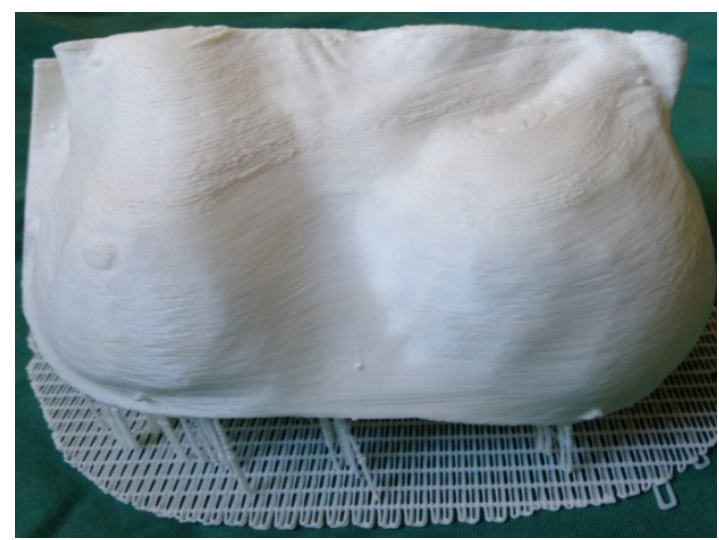

Figure 4: A three dimensional (3D)-printed haptic, tactile biomodel of the breast (same patient as Figure 1), produced from computed tomography (CT) data. Printing performed with the Cube 2 printer (3D Systems, Rock Hill, SC, USA) using Cubify software (3D Systems), and using white polylactic acid (PLA) filament ink. Reproduced with permission from: Chae MP, Hunter-Smith DJ, Spychal RT, Rozen WM. 3D Volumetric Analysis for Planning Breast Reconstructive Surgery. Breast Cancer Res Treat. 2014 June; [Epub ahead of Print].

\section{Discussion}

Volumetric analysis can potentially provide an useful information for the breast reconstructive surgeons during preoperative planning, but as yet, an accurate, reproducible, objective method of assessing volume has been elusive [6]. Most recently developed imaging technique, the 3D photography, fails to capture the posterior surface of the breasts and fails to account for individual variances in the chest wall anatomy [2]. We describe a relatively straightforward, accurate, reproducible method of evaluating breast volume at no cost using Osirix (Pixmeo, Geneva, Switzerland), a free third-party software. By highlighting the region of interest from each transverse slice of the CT scan, the software calculates the volume. Using this technique, we could derive the volume difference of $124.61 \mathrm{~cm}^{3}$ in our illustrated case of breast asymmetry. Interestingly, we also calculated the volume of the implant in the left breast $\left(321.47 \mathrm{~cm}^{3}\right)$, which is the same as the volume of the implant inserted according to our records $(320 \mathrm{~g})$. Furthermore, using a 3D software, such as Magics (Materialise, Leuven, Belgium), we have produced a $3 \mathrm{D}$ visualization representing the volume difference.

While multiple techniques for assessing breast volume have been described, most are limited by accuracy, invasiveness or differentiation between breast components (skin envelope, parenchyma, implant, chest wall). 3D photography only takes into account surface area and surface contour, external prostheses can be inaccurate, and volume displacement techniques are uncomfortable or invasive. A recent manuscript described surface landmark techniques for assessing breast volumes (sternal notch-to-nipple, inframammary fold-to-nipple, and inframammary fold-to-fold projection distances) [17]. Our technique eliminates such inaccuracy by being able to eliminate chest wall deformities in any measurements, and provide an objective assessment of parenchymal volume.

Preoperative planning with CTA has enabled physicians to select the donor site, the perforators, and design flaps, which has led to significantly improved clinical outcomes and reduced complications $[1,3,7,9,10,18]$. In order to interpret CTA, the user is limited to the data being represented on a $2 \mathrm{D}$ computer screen. The recent introduction of RP and biomodeling for medical applications has yielded 3D haptic biomodels that can be held in the clinician's hands [4]. They provide a tactile feedback in addition to creating an accurate representation of the spatial relationship between anatomical structures. The $3 \mathrm{D}$ printing technology has made the fabrication of haptic biomodels more accessible and affordable while maintaining accurate anatomical details $[4,12,15]$. Our described technique has not yet been evaluated in a patient or correlated with patient outcomes. However, in this current study, we demonstrate that our approach is a valuable, reproducible, and affordable addition to preoperative planning in breast reconstruction.

\section{Patient consent}

Written consent for the use of this patient's images was provided by the patient.

\section{Declarations}

The content of this article has not been submitted or published elsewhere. There was no source of funding for the article. The authors declare that there is no source of financial or other support, or any financial or professional relationships, which may pose a competing interest. All authors contributed to the preparation of this manuscript and are in agreement with the content of the manuscript. 
Citation: Hunter-Smith DJ, Alexandra R, Spychal RT, Michael PC (2015) 3D Volumetric Analysis and Haptic Modeling for Preoperative Planning in Breast Reconstruction. Anaplastology 4: 138. doi:10.4172/2161-1173.1000138

Page 4 of 4

\section{References}

1. Rozen WM, Chubb D, Grinsell D, Ashton MW (2011) Computed tomographic angiography: clinical applications. Clin Plast Surg 38: 229-239.

2. Chae MP, Hunter-Smith DJ, Spychal RT, Rozen WM (2014) 3D volumetric analysis for planning breast reconstructive surgery. Breast Cancer Res Treat 146: 457-460.

3. Rozen WM, Ashton MW, Stella DL, Phillips TJ, Grinsell D and others (2008) The accuracy of computed tomographic angiography for mapping the perforators of the deep inferior epigastric artery: a blinded, prospective cohort study.Plast Reconstr Surg 122 1003-1009.

4. Chae MP, Hunter-Smith DJ, Rozen WM (2014) Image-guided 3Dprinting and haptic modeling in plastic surgery. In: Saba L, Rozen WM, Alonso-Burgos A, Ribuffo D, editors. Imaging in plastic surgery. London, UK: CRC Taylor and Francis Press.

5. Tepper OM, Small K, Rudolph L, Choi M, Karp N (2006) Virtual 3dimensional modeling as a valuable adjunct to aesthetic and reconstructive breast surgery. Am J Surg 192: 548-551.

6. Galdino GM, Nahabedian M, Chiaramonte M, Geng JZ, Klatsky S, et al. (2002) Clinical applications of three-dimensional photography in breast surgery. Plast Reconstr Surg 110: 58-70.

7. Rozen WM, Ashton MW, Grinsell D, Stella DL, Phillips TJ, et al. (2008) Establishing the case for CT angiography in the preoperative imaging of abdominal wall perforators. Microsurgery 28: 306-313.

8. Rozen WM, Phillips TJ, Stella DL, Ashton MW (2009) Preoperative CT angiography for DIEP flaps: 'must-have' lessons for the radiologist. J Plast Reconstr Aesthet Surg 62: 650-651.

9. Rozen WM, Anavekar NS, Ashton MW, Stella DL, Grinsell D, et al. (2008) Does the preoperative imaging of perforators with CT angiography improve operative outcomes in breast reconstruction? Microsurgery 28: 516-523.

10. Dionyssiou D, Demiri E, Tsimponis A, Boorman J (2014) Predesigned breast shaping assisted by multidetector-row computed tomographic angiography in autologous breast reconstruction. Plast Reconstr Surg 133: $100-108$.

11. Agrawal MD, Thimmappa ND, Vasile JV, Levine JL, Allen RJ, et al. (2014) Autologous Breast Reconstruction: Preoperative Magnetic Resonance Angiography for Perforator Flap Vessel Mapping.J Reconstr Microsurg.

12. Gerstle TL, Ibrahim AM, Kim PS, Lee BT, Lin SJ (2014) A plastic surgery application in evolution: three-dimensional printing. Plast Reconstr Surg 133: 446-451.

13. Rozen WM, Ting JW, Baillieu C, Leong J (2012) Stereolithographic modeling of the deep circumflex iliac artery and its vascular branching: a further advance in computed tomography-guided flap planning.Plast Reconstr Surg 130: 380e-382e.

14. Rozen WM, Ting JW, Leung M, Wu T, Ying D and others (2012) Advancing image-guided surgery in microvascular mandibular reconstruction: combining bony and vascular imaging with computed tomography-guided stereolithographic bone modeling. Plast Reconstr Surg 130: 227-229.

15. Cohen A, Laviv A, Berman P, Nashef R, Abu-Tair J (2009) Mandibular reconstruction using stereolithographic 3-dimensional printing modeling technology. Oral Surg Oral Med Oral Pathol Oral Radiol Endod 108: 661-666.

16. Way TP, Barner KE;(1997);Automatic visual to tactile translation--Part II: Evaluation of the TACTile Image Creation System.IEEE Trans Rehabil Eng 5: 95-105.

17. Longo B, Farcomeni A, Ferri G, Campanale A, Sorotos M, et al. (2013) The BREAST-V: a unifying predictive formula for volume assessment in small, medium, and large breasts. Plast Reconstr Surg 132: 1-7.

18. Rozen WM, Ashton MW, Whitaker IS, Wagstaff MJ, Acosta R (2009) The financial implications of computed tomographic angiography in DIEP flap surgery: a cost analysis. Microsurgery 29: 168-169. 